\title{
SEVERE INFECTIONS IN PATIENTS WITH SYSTEMIC AUTOIMMUNE MYOPATHIES: A RETROSPECTIVE COHORT STUDY
}

\author{
Leonardo Santos Hoff ${ }^{1}$, Rafael Alves Cordeiro ${ }^{1}$, Samuel Katsuyuki Shinjo1,* \\ 1. Faculdade de Medicina FMUSP, Universidade de São Paulo, São Paulo (SP), Brazil. \\ *Corresponding author: samuel.shinjo@usp.br
}

\section{BACKGROUND}

Patients with systemic autoimmune myopathies (SAMs) are at higher risk of infections either by the disease manifestations or by the immunosuppressive treatment, and infections represent a major cause of death. This study aims to evaluate the characteristics and risk factors for severe infections in patients with SAMs.

\section{METHODS}

This is a single-center retrospective cohort study, from 2001 to 2017, that included consecutive adult patients with SAMs (dermatomyositis, polymyositis, amyopathic dermatomyositis, and immune-mediated necrotizing myopathies). Severe infections were defined as any infection requiring parenteral therapy or tuberculosis. Cox multivariate regression analysis was used to estimate hazard ratios (HR) with a 95\% confidence interval (Cl) for clinical and treatment features associated with infections.

\section{RESULTS}

Eighty-one (31.2\%) out of 260 patients had a severe infection. These infected patients were older ( $47.5 \pm 16.3$ vs. $41.7 \pm 13.9$ years; $p=0.009)$, more of the male sex ( $37.0 \%$ vs. $19.0 \%, p=0.002)$, had more severe proximal limb weakness $(45.7 \%$ vs. $30.2 \% ; p=0.015$ ), more skin ulcers ( $28.4 \%$ vs. $10.6 \% ; p<0.001)$, lower median serum levels of creatine phosphokinase (980 $[\mathrm{IQR}=169,4311]$ vs. $2396[393,8000] \mathrm{U} / \mathrm{L} ; \mathrm{p}=0.008)$, were less exposed to methotrexate $(70.4 \% \mathrm{vs} .84 .4 \% ; p=0.009)$, but received more intravenous immunoglobulin ( $56.8 \%$ vs. $34.1 \%$; $p=0.001$ ). A total of 155 severe infections events occurred: $33(21.3 \%$ ) cutaneous infectious, 32 (20.6\%) pneumonias, 28 (18.1\%) aspiration pneumonias, $14(9.0 \%)$ pyelonephritis, 10 (6.5\%) tuberculosis, and 38 (24.5\%) other. The main pharmacological therapy at infection onset was glucocorticoids (84.5\%), and overall lethality was $15.5 \%$. The multivariate Cox analysis revealed male sex $(\mathrm{HR}=1.84 ; 95 \% \mathrm{Cl}=1.11-3.07)$, severe proximal weakness $(\mathrm{HR}=2.23$, $95 \% \mathrm{Cl}=1.20-4.13)$, and skin ulcers $(\mathrm{HR}=2.23 ; 95 \% \mathrm{Cl}=1.26-3.93)$ as risk factors for severe infections, whereas serum levels of creatine phosphokinase $>5000 \mathrm{U} / \mathrm{L}(\mathrm{HR}=0.42 ; 95 \% \mathrm{Cl}=0.21-0.83)$, methotrexate $(\mathrm{HR}=0.22 ; 95 \% \mathrm{Cl}=0.13-0.38)$, and azathioprine $(\mathrm{HR}=0.16 ; 95 \% \mathrm{Cl}=0.09-0.29)$ as protective factors. Methotrexate and azathioprine remained protective after a sensitivity analysis.

\section{CONCLUSION}

Patients with SAMs have a high incidence of severe infections, which have a $15.5 \%$ of lethality. The protective effect of methotrexate and azathioprine may be explained by a sparing effect over glucocorticoid, which was the most used drug during an acute infection. Rheumatologists should be aware of the main types of infections and the features associated with them, in an attempt to improve the outcomes and the care of patients with SAMs.

\section{KEYWORDS}

Infection, Pneumonia, Systemic autoimmune myopathy, Polymyositis, Dermatomyositis. 